\title{
Atomistic computer simulations of the cement degradation mechanisms in the context of geological carbon sequestration
}

\author{
SYLVIA M. MUTISYA ${ }^{1}$ AND ANDREY G. KALINICHEV ${ }^{2}$ \\ ${ }^{1}$ Institute Mines-Telecom Atlantique \\ ${ }^{2}$ IMT Atlantique \\ Presenting Author: mutisya@subatech.in2p3.fr
}

One of the major concerns of many subsurface operations, such as geological $\mathrm{CO}_{2}$ sequestration, is the degradation of wellbore cement and the potential fluid migration to the surface through leakage pathways. While leakage can occur in wells due to faulty construction and other mechanical defects, geochemical reactions induced by the injected fluids could also cause cement degradation, damaging the wells and leading to the leaks. Cement is highly alkaline $(\mathrm{pH}>12.5)$, and acidic $\mathrm{CO}_{2}$-rich fluids $(\mathrm{pH}<6)$ are thermodynamically incompatible with it. Thus, exposure of cement to $\mathrm{CO}_{2}$-saturated fluids lowers the $\mathrm{pH}$ of the cement pore fluid, which may lead to failure of operations due to concrete degradation. To better understand these processes, we focus here on identifying and quantitatively characterizing on the fundamental molecular scale possible cement degradation mechanisms and reaction pathways, fluid transport rate and the geochemical variables that affect fluid-cement interactions.

We use biased ab-initio molecular dynamics (AIMD) simulations to explore the reactivity of $\mathrm{CO}_{2}$ with the basal and edge surfaces of the portlandite cement phase in $\mathrm{scCO}_{2}$ and water-rich conditions. The metadynamics approach is applied to accelerate the dynamics of the rare reaction events and to investigate their mechanisms. Our simulations show that supercritical $\mathrm{CO}_{2}$ undergoes a rapid barrierless carbonation reaction with the edge surfaces of portlandite crystals. However, the carbonation reaction soon ceases due to the deposition of (bi)carbonate surface complexes which form a carbonate layer. On the other hand, the presence of water alters the interaction of $\mathrm{CO}_{2}$ with the portlandite surfaces, as $\mathrm{H}_{2} \mathrm{O}$ molecules form wellstructured aqueous surface layers. Thus, the water content within the portlandite pores is the rate limiting step in the carbonation reaction of portlandite with $\mathrm{H}_{2} \mathrm{O} / \mathrm{CO}_{2}$ fluid. As such, $\mathrm{CO}_{2}$ reactivity for pores with highly structured water surface layers (with no bulk-like water) is expected to be limited due to the attenuated inward diffusion of the $\mathrm{CO}_{2}$ molecules. Dissolution of calcium atoms can be expected to enhance the carbonation reaction by interacting with the dissolved (bi)carbonate/carbonic acid to form calcite in the presence of water. However, no such dissolution events were observed in our simulations. 